\title{
DESENVOLVIMENTO E REPRODUÇÃO DE Dirphia moderata (LEPIDOPTERA: SATURNIIDAE) EM Eucalyptus cloeziana E Psidium guajava EM LABORATÓRIO ${ }^{1}$
}

\author{
Fabrício Fagundes Pereira², Antonio José Vinha Zanuncio ${ }^{3}$, João Paulo de Mello Felipe ${ }^{4}$, Alexandre \\ Simões Lorenzon ${ }^{3}$ e Glauco da Cruz Canevari ${ }^{2}$
}

\begin{abstract}
RESUMO - Neste trabalho, estudaram-se o desenvolvimento e a reprodução de Dirphia moderata Bouvier, 1919 (Lepidoptera: Saturniidae) alimentada com Eucalyptus cloeziana F. Muell ou Psidium guajava L. Os períodos de pré-oviposição, oviposição e incubação e a viabilidade e número de ovos por fêmea foram de $6,71 \pm 0,18 \mathrm{dia} ; 4,00 \pm 0,66 \mathrm{dia} ; 18,14 \pm 0,18 \mathrm{dia} ; 17,54 \pm 3,78 \% ; 121,71 \pm 18,96 \%$ para esse inseto com E. cloeziana e de $3,86 \pm 0,26 \mathrm{dia} ; 5,86 \pm 0,40 \mathrm{dia} ; 17,71 \pm 0,21 \mathrm{dia} ; 23,12 \pm 10,81 \% ; 112,00 \pm 8,79 \% \mathrm{com}$ P. guajava, respectivamente. A duração e viabilidade larval (\%) foram de 53,00 $\pm 0,09$ e 80,00 $\pm 0,99$ e de $55,83 \pm 0,29$ e de $77,5 \pm 6,60$ para lagartas de $D$. moderata criadas nos primeiro e segundo hospedeiros, respectivamente. A duração e viabilidade das fases de pré-pupa e pupa foram de 4,84 $\pm 0,07$ dia e $96,88 \pm$ $3,13 \%$ e de $37,64 \pm 1,20$ dia e $84,37 \pm 6,53 \%$ com E. cloeziana e de 3,82 $\pm 0,06$ dia e $100 \%$ e de 49,93 $\pm 2,04$ dias e 75,00 $\pm 7,79 \%$ com $P$. guajava, respectivamente. A razão sexual de D. moderata foi de 0,48 e 0,46 e a longevidade, de 6,78 e 8,84 dias para machos e de 10,23 e 10,07 dias para fêmeas desse inseto com E. cloeziana e $P$. guajava, respectivamente. D. moderata apresentou melhor desenvolvimento e reprodução em E. cloeziana, mas $P$. guajava pode, também, ser utilizado para a criação desse inseto em laboratório.
\end{abstract}

Palavras-chave: Desfolhadores de eucalipto, Saturniidae e eucalipto.

\section{DEVELOPMENT AND REPRODUCTION OF Dirphia moderata (LEPIDOPTERA: SATURNIIDAE) ON Eucalyptus cloeziana OR Psidium guajava LEAVES UNDER LABORATORY CONDITIONS}

\begin{abstract}
This work studied the development and reproduction of Dirphia moderata Bouvier, 1919 (Lepidoptera: Saturniidae) fed on Eucalyptus cloeziana F. Muell or Psidium guajava L. The periods of pre-oviposition, oviposition and egg incubation as well as viability and number of eggs per female were $6.71 \pm 0.18$ days; $4.00 \pm 0.66$ days; $18.14 \pm 0.18$ days; $17.54 \pm 3.78 \% ; 121.71 \pm 18.96$ for this insect on E. cloeziana and $3.86 \pm 0.26$ days; $5.86 \pm 0.40$ days; $17.71 \pm 0.21$ days; $23.12 \pm 10.81 \% ; 112.00 \pm 8.79$ on $\boldsymbol{P}$. guajava, respectively. Larva stage duration and viability (\%) were $53.00 \pm 0.09$ and $80.00 \pm 0.99$ and $55.83 \pm 0.29$ and $77.5 \pm 6.60$ for caterpillars of $\boldsymbol{D}$. moderata reared with the first and second hosts, respectively. Duration and viability of the pre-pupa and pupa stages were $4.84 \pm 0.07$ days and $96.88 \pm 3.13 \%$ and $37.64 \pm 1.20$ days and $84.37 \pm 6.53 \%$ on E. cloeziana and $3.82 \pm 0.06$ days and $100.00 \%$ and $49.93 \pm 2.04$ days and $75.00 \pm 7.79 \%$ on P. guajava, respectively. Sex rate of D. moderata was 0.48 and 0.46 and longevity was 6.78 and 8.84 days for males and 10.23 and 10.07 days for females when this insect fed on E. cloeziana and $\boldsymbol{P}$. guajava, respectively. Dirphia moderata presented better development and reproduction with $\boldsymbol{E}$. cloeziana but $\boldsymbol{P}$. guajava can also be used to rear this insect in the laboratory.
\end{abstract}

Keywords: Eucalyptus defoliator, Saturniidae and Eucalypt.

\footnotetext{
${ }^{1}$ Recebido em 15.05.2007 e aceito para publicação em 22.08.2008.

${ }^{2}$ Departamento de Biologia Animal/BIOAGRO da Universidade Federal de Viçosa (UFV). E-mail: <ffpereira@insecta.ufv.br> e<glaucocanevari@yahoo.com.br>.

${ }^{3}$ Graduação em Engenharia Florestal da UFV.

${ }^{4}$ Departamento de Agronomia da Universidade Federal de Viçosa - UFV
} 


\section{INTRODUÇÃO}

O gênero Eucalyptus apresenta mais de 600 espécies adaptadas a diferentes climas e solos (HIGA, 2003), e suas espécies são danificadas por insetos de hospedeiros nativos da flora brasileira, especialmente da família Myrtaceae (ZANUNCIO et al., 2001). Insetos, alimentando-se de hospedeiros filogeneticamente próximos (ZANUNCIO, et al., 1993), podem causar danos em reflorestamentos com eucalipto por estarem em processo de adaptação a essa planta (SANTOS et al., 1989; ZANUNCIO et al., 1990), sendo os lepidópteros desfolhadores os mais importantes (ZANUNCIO et al., 1998).

Os lepidópteros desfolhadores de eucalipto (GUEDES, et al., 2000) incluem espécies do gênero Dirphia Hubner (1819) que, embora consideradas pragas secundárias, merecem atenção. Dirphia $\mathrm{sp}$. foi registrada em Eucalyptus saligna Sm na região de Piracicaba, São Paulo (BRANGANÇA, et al., 1998; BITTENCOURT et al., 2003), e Dirphia avia (Stoll, 1780) e Dirphiopsis trisignata (Felder e Felder, 1874) (Saturniidae) em eucaliptais em Caçapava e São José dos Campos, São Paulo. Alguns aspectos biológicos de Cerodirphia (Dirphia) rosacordis (Walker, 1855) (Saturniidae) foram estudados em eucalipto (ZANUNCIO et al., 1992). Além disso, essa última espécie foi relatada em 700 ha, com desfolha total de 300 ha de Eucalyptus grandis Hill ex Maiden e E. saligna na região de Buritizeiro, Minas Gerais (ZANUNCIO, 1993). Dirphia moderata Bouvier, 1919 (Saturniidae) (PEREIRA et al., 2008), coletada em Montes Claros, Minas Gerais, foi criada em Eucalyptus urophylla (ZANUNCIO et al., 1994) e relatada como D. avicula, sendo essa sinonímia corrigida após uma revisão desse gênero. Uma espécie próxima, Pseudodirphia (Dirphia) eumedidoides (Vuillot, 1892), foi estudada (SANTOS et al., 1993). Dirphia moderata foi relatada em Anacardium occidentale L., Schinus terebinthifolius (Raddi) e Rapanea umbellata Mez. (DIAS, 1988). Dirphia araucariae Jones, 1908, Dirphia baroma (Schaus, 1906), Dirphia fornax (Druce, 1903), Dirphia muscosa Schaus, 1898 e Dirphia ursina Walker, 1855 foram capturadas com armadilhas luminosas e pano iluminado em regiões fisiográficas do Rio Grande do Sul (CORSEUIL et al., 2002).

Testes preliminares em laboratório revelaram o elevado potencial biótico de D. moderata em folhas de eucalipto e goiabeira, o que indica que essa espécie pode ter plantas de mirtáceas como hospedeiros originais e estar se adaptando às espécies de eucalipto, a exemplo de outros lepidópteros desfolhadores como Psorocampa denticulata Schaus (Notodontidae) (SANTOS et al., 1982) e Apatelodes sericea Schaus, 1896 (Eupterotidae) (SANTOS et al., 1985), que, apesar de consideradas espécies secundárias, passaram à condição de pragas primárias. Isso justifica estudos para determinar aspectos biológicos de $D$. moderata em goiabeira, por ser uma mirtácea nativa, possivelmente utilizada por esse lepidóptero desfolhador, antes da introdução do eucalipto no Brasil. Portanto, o objetivo deste trabalho foi estudar o desenvolvimento e reprodução de $D$. moderata com folhas de eucalipto (Eucalyptus cloeziana F. Muell.) ou de goiabeira (Psidium guajava L.) em laboratório.

\section{MATERIAL E MÉTODOS}

Posturas de $D$. moderata foram obtidas de colônia mantida no Laboratório de Controle Biológico de Insetos do Departamento de Biologia Animal da Universidade Federal de Viçosa (UFV) e acondicionada em placas de Petri ( $9,0 \mathrm{~cm}$ de diâmetro por $1,2 \mathrm{~cm}$ de altura), com um chumaço de algodão umedecido com água destilada no seu interior e mantidas a $25 \pm 2^{\circ} \mathrm{C}$, umidade relativa de $70 \pm 10 \%$ e fotofase de $12 \mathrm{~h}$. Dois lotes com 40 lagartas cada um, imediatamente após a eclosão, foram obtidos para estudo dos parâmetros biológicos de D. moderata em substrato de eucalipto ou de goiabeira. Grupos de cinco lagartas foram colocados por pote plástico (500 $\mathrm{mL}$ ), com a tampa telada no centro. Isso foi necessário, pois $D$. moderata apresenta hábito gregário que, se modificado, poderia interferir no seu desempenho biológico. Um tubo de vidro, tipo anestésico, com água e folhas de E. cloeziana ou de P. guajava, com os pecíolos envoltos por algodão, foi colocado no interior de cada pote e trocado diariamente. No quarto estádio, em razão do tamanho, as lagartas foram transferidas para gaiolas teladas de $30 \times 30 \times 30 \mathrm{~cm}$, com fundo de madeira e tampas de vidro. Pupas obtidas foram colocadas em potes plásticos (500 $\mathrm{mL}$ ) com terra umedecida. Casais de D. moderata, ao emergirem, foram colocados em gaiolas semelhantes com galhos de E. cloeziana ou de P. guajava em chumaço de algodão para obtenção de ovos desse inseto. O período embrionário e a viabilidade de ovos, o número de estádios, o período e viabilidade das fases de larva, de pré-pupa e de pupa, além da razão sexual, da longevidade de adultos e da capacidade de postura de $D$. moderata, foram obtidos.

A razão de crescimento em insetos pode ser prevista por regras empíricas com a cápsula cefálica de lagartas crescendo em progressão geométrica e aumentando 
em largura a cada ecdise numa razão constante (média de 1,4$)$. Isso torna possível deduzir o número de ecdises de insetos (Dyar 1890). Por isso, o número e duração de cada estádio de $D$. moderata foram obtidos pela medição diária da cápsula cefálica de suas lagartas em microscópio estereoscópico e ocular micrométrica. Os dados foram analisados pelo teste $\mathrm{F}$ a $5 \%$ de significância. Adultos dessa mariposa foram montados, fotografados e enviados ao Departamento de Zoologia, Setor de Ciências Biológicas, da Universidade Federal do Paraná, Curitiba, Estado do Paraná, Brasil, para identificação e depositados no Museu de Entomologia da Universidade Federal de Viçosa (UFV).

\section{RESULTADOS E DISCUSSÃO}

\subsection{Ovos}

Os períodos (dias) de pré-oviposição e de oviposição, de incubação, de viabilidade de ovos (\%) e número de ovos por fêmea de $D$. moderata foram de $6,71 \pm 0,18$; $4,00 \pm 0,66 ; 18,14 \pm 0,18 ; 17,54 \pm 3,78$ e $121,71 \pm 18,96$ com E. cloeziana e de 3,86 $\pm 0,26 ; 5,86 \pm 0,40 ; 17,71 \pm$ 0,$21 ; 23,12 \pm 10,81 ;$ e $112,00 \pm 8,79$ com $P$. guajava, respectivamente, sem diferença entre hospedeiros, exceto no período de oviposição, que foi menor em E. cloeziana, fato de pouca importância devido ao número semelhante de ovos nos dois hospedeiros (Quadro 1). Abaixa viabilidade de ovos de D. moderata com E. cloeziana ou P. guajava pode ser atribuída ao fato de se terem individualizados os casais desse inseto em gaiolas para facilitar a coleta dos dados, o que descartou a possibilidade de acasalamento com composição variada de parceiros.

Essa afirmação pode ser suportada pelo fato de que, na colônia de $D$. moderata mantida nesse laboratório, vários adultos eram colocados numa mesma gaiola para se acasalarem, e a viabilidade dos ovos nessas circunstâncias estava sempre acima de $70 \%$. Isso evidencia que acasalamentos múltiplos podem aumentar o potencial reprodutivo de insetos gregários como relatado para Hylesia nanus (Walker, 1855) (Lepidoptera: Attacidae), que apresentou melhor desempenho reprodutivo, com três casais em uma mesma gaiola (SANTOS et al., 1988). No entanto, $70 \%$ dos ovos de casais individualizados de D. avicula (=D. moderata), originados de lagartas criadas com Eucalyptus urophylla S.T. Blake, foram férteis (ZANUNCIO et al., 1994), o que pode indicar que essa espécie seja um hospedeiro melhor para $D$. avicula que E. cloeziana ou P. guajava. Isso pode ser devido a barreiras químicas de plantas que atuam, principalmente, na redução da digestibilidade dos insetos e afetam seu desenvolvimento na atual e nas próximas gerações (MAURICIO e RAUSHER, 1997).

\subsection{Larvas}

A duração (dias) e viabilidade larval (\%) de $D$. moderata foram de 53,00 $\pm 0,09$ e 80,00 $\pm 0,99$ e 55,83 $\pm 0,29$ e 77,50 $\pm 6,60$ para lagartas dessa espécie criadas com E. cloeziana e P. guajava, respectivamente (Quadro 1), com seis estádios nos dois hospedeiros (Figura 1). A menor duração do período larval de $D$. moderata com E. cloeziana evidencia que esse hospedeiro seja mais adequado para o desenvolvimento desse inseto. No entanto, a viabilidade dessa fase foi semelhante nos dois hospedeiros, o que demonstra que $D$. moderata se desenvolve, também, com $P$. guajava. Isso pode ser reforçado pelo fato de esse inseto ter sido criado com E. cloeziana ou P. guajava por mais de uma geração, o que sugere ter havido adaptação alimentar das lagartas, que foram retiradas de plantas de eucalipto e criadas em folhas de goiabeira. No entanto, espécies desfolhadoras de eucalipto podem ter melhor desenvolvimento em hospedeiros nativos, pois Thyrinteina arnobia (Stoll, 1782) (Geometridae), criada por 14 gerações em folhas de E. grandis ou $P$. guajava, apresentou melhor desenvolvimento e reprodução com esse último hospedeiro (OLIVEIRA et al., 2005). Thyrinteina arnobia e outras espécies de insetos se encontram em processo de adaptação a plantas de eucalipto ao fugirem da pressão exercida por barreiras físicas e químicas que podem existir nas mirtáceas nativas brasileiras (HOLTZ et al., 2003b).

\subsection{Pupas}

A duração (dias) e viabilidade (\%) das fases de pré-pupa e de pupa de $D$. moderata foram de 4,84 $\pm 0,07$ e $96,88 \pm 3,13$ e de $37,64 \pm 1,20$ e $84,37 \pm 6,53$ com E. cloeziana e de 3,82 $\pm 0,06$ e 100,00 e de 49,93 $\pm 2,04$ e 75,00 \pm 7,79 com $P$. guajava, respectivamente, com influência do substrato apenas, no período pupal, menor com folhas de E. cloeziana, o que indica adaptação desse lepidóptero a essa espécie de eucalipto. entretanto, a maior duração dessa fase para T. arnobia em E. cloeziana, que em P. guajava, pode ser devido ao fato de essa espécie ser mais comum na última planta, o que possibilita evitar a atuação de mecanismos de defesa desse hospedeiro (defesas químicas que interferem negativamente no desempenho do inseto) (HOLTZ et al., 2003ab).

R. Árvore, Viçosa-MG, v.32, n.6, p.1119-1124, 2008 
Quadro 1 - Aspectos biológicos de Dirphia moderata (Lepidoptera: Saturniidae) com Eucalyptus cloeziana ou Psidium guajava a $25 \pm 2{ }^{\circ} \mathrm{C}, 70 \pm 10 \%$ de umidade relativa e fotofase de $12 \mathrm{~h}$

Table 1 - Biological aspects of Dirphia moderata (Lepidoptera: Saturniidae) fed on Eucalyptus cloeziana or Psidium guajava leaves at $25 \pm 2{ }^{\circ} \mathrm{C}, 70 \pm 10 \%$ relative humidity and photo phase of 12 hours

\begin{tabular}{lccrc}
\hline Aspectos Biológicos & $\begin{array}{c}\text { Eucalyptus cloeziana } \\
\text { (Média } \pm \text { erro-padrão) }\end{array}$ & (n) & $\begin{array}{r}\text { Psidium guajava } \\
\text { (Média } \pm \text { erro-padrão) }\end{array}$ & (n) \\
\hline Pré-oviposição (dias) & $6,71 \pm 0,18 \mathrm{a}$ & 7 & $3,86 \pm 0,26 \mathrm{a}$ & 7 \\
Oviposição (dias) & $4,00 \pm 0,66 \mathrm{~b}$ & 7 & $5,86 \pm 0,40 \mathrm{a}$ & 7 \\
Total de ovos/fêmea (unidades) & $121,71 \pm 18,96 \mathrm{a}$ & 7 & $112,00 \pm 8,79 \mathrm{a}$ & 7 \\
Período de incubação (dias) & $18,14 \pm 0,18 \mathrm{a}$ & 7 & $17,71 \pm 0,21 \mathrm{a}$ & 7 \\
Viabilidade de ovos (\%) & $17,54 \pm 3,78 \mathrm{a}$ & 7 & $23,12 \pm 10,81 \mathrm{a}$ & 7 \\
Duração de fase larval (dias) & $53,00 \pm 0,09 \mathrm{a}$ & 32 & $55,83 \pm 0,29 \mathrm{~b}$ & 32 \\
Viabilidade de fase larval (\%) & $80,00 \pm 0,99 \mathrm{a}$ & 40 & $77,50 \pm 6,60 \mathrm{a}$ & 40 \\
Duração de fase de pré-pupa (dias) & $4,84 \pm 0,07 \mathrm{a}$ & 32 & $3,82 \pm 0,06 \mathrm{a}$ & 32 \\
Viabilidade de fase de pré-pupa (\%) & $96,88 \pm 3,13 \mathrm{a}$ & 32 & $100,00 \pm 0,00 \mathrm{a}$ & 32 \\
Duração da fase de pupa (dias) & $37,64 \pm 1,20 \mathrm{a}$ & 32 & $49,93 \pm 2,04 \mathrm{~b}$ & 32 \\
Viabilidade da fase de pupa (\%) & $84,37 \pm 6,53 \mathrm{a}$ & 32 & $75,00 \pm 7,79 \mathrm{a}$ & 32 \\
\hline
\end{tabular}

${ }^{(1)}$ Médias seguidas de mesma letra minúscula por linha não diferem entre si, pelo teste $\mathrm{F}(\mathrm{P}=0,05)$.

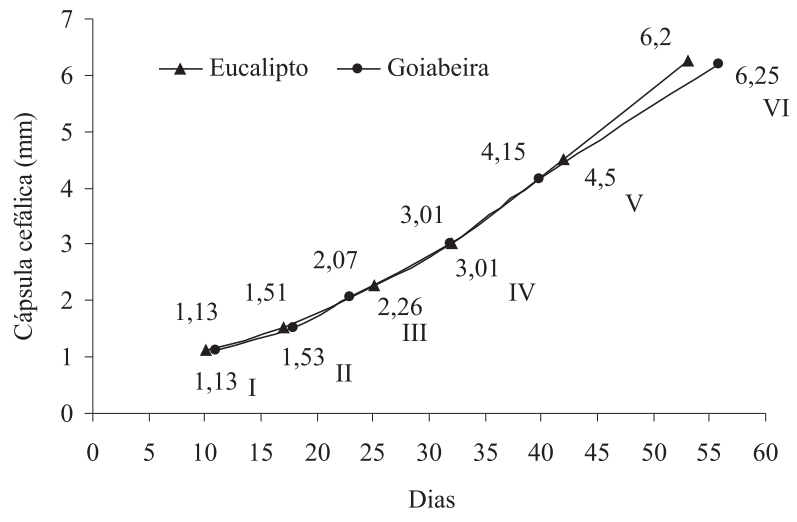

Figura 1 - Largura da cápsula cefálica e duração dos estádios de Dirphia moderata (Lepidoptera: Saturniidae) com Eucalyptus cloeziana e Psidium guajava a $25 \pm 2^{\circ} \mathrm{C}$, $70 \pm 10 \%$ de umidade relativa e fotofase de $12 \mathrm{~h}$.

Figure 1 - Width of the head capsule and duration of the instars of Dirphia moderata (Lepidoptera: Saturiniidae) fed on Eucalyptus cloeziana or Psidium guajava at $25 \pm 2{ }^{\circ} \mathrm{C}, 70 \pm 10 \%$ relative humidity and photo phase of 12 hours.

\subsection{Adultos}

A longevidade (dias) de D. moderata foi semelhante com 10,23 e 10,07 dias para fêmeas desse lepidóptero criado com E. cloeziana e $P$. guajava, respectivamente, mas machos dessa mariposa foram mais longevos (8,84 dias) em $P$. guajava (Figura2).Apesarde folhas de E. cloeziana terem proporcionado melhores condições de desenvolvimento para D. moderata, a maior duração da fase larval de indivíduos que originaram machos em $P$. guajava pode permitir maior acúmulo de energia e gerar adultos mais longevos.

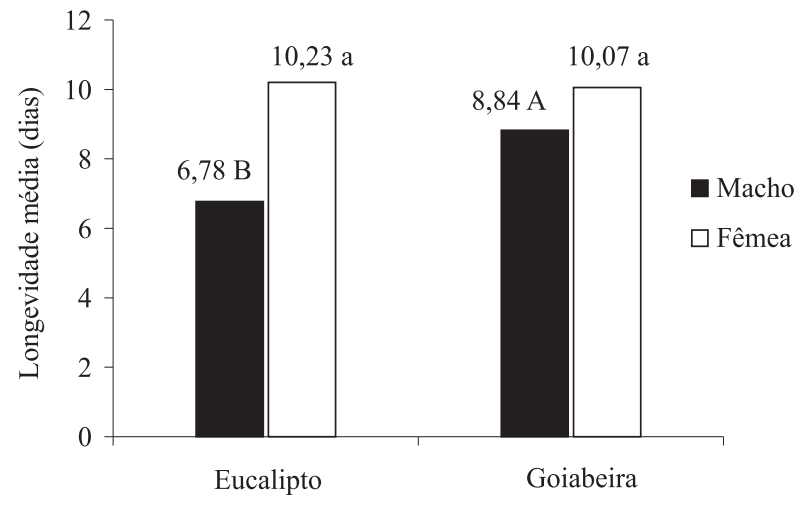

Figura 2 - Longevidade de adultos de Dirphia moderata (Lepidoptera: Saturniidae) de lagartas alimentadas com Eucalyptus cloeziana ou Psidium guajava a $25 \pm 2{ }^{\circ} \mathrm{C}, 70 \pm 10 \%$ de umidade relativa e fotofase de $12 \mathrm{~h}$. Médias nos tratamentos com mesma letra maiúscula ou minúscula não diferem entre si, pelo teste $\mathrm{F}(\mathrm{P} \leq 0,05)$.

Figure 2 - Longevity of Dirphia moderata (Lepidoptera: Saturniidae) adults from larvae fed on Eucalyptus cloeziana or Psidium guajava at $25 \pm 2^{\circ} \mathrm{C}, 70$ $\pm 10 \%$ relative humidity and photo phase of 12 hours. Means followed by the same capital or lowercase letters per treatment do not differ, using the F test $(P \leq 0.05)$.

A razão sexual de $D$. moderata foi semelhante, com 0,48 e 0,46, em E. cloeziana e P. guajava, respectivamente. A razão sexual desse inseto foi, também, de 0,48 em E. urophylla, o que demonstra que espécies de eucalipto não alteram esse aspecto biológico (ZANUNCIO et al., 1994). 
Dirphia moderata pode ser criada em E. cloeziana ou P. guajava em laboratório por várias gerações. Isso permite conhecer seus aspectos biológicos e comportamentais que podem ser utilizados para elaboração de planos de manejo, caso ocorram surtos populacionais dessa espécie em plantios de eucalipto ou de goiabeira. De maneira geral, D. moderata apresentou melhor desenvolvimento e reprodução em E. cloeziana, mas $P$. guajava pode, também, ser utilizado na criação desse inseto em laboratório.

\section{AGRADECIMENTOS}

Ao Conselho Nacional de Desenvolvimento Científico e Tecnológico (CNPq), à Coordenação de Aperfeiçoamento de Pessoal de Nível Superior (CAPES) e à Fundação de Amparo à Pesquisa do Estado de Minas Gerais (FAPEMIG), pelo apoio financeiro; e ao Dr. Olaf Hermann Hendrik Mielke, do Departamento de Zoologia da Universidade Federal do Paraná, pela identificação de Dirphia moderata.

\section{REFERÊNCIAS}

BITTENCOURT, M. A. et al. Fauna de Lepidoptera associada a um ecossistema natural no estado de São Paulo. Arquivos do Instituto Biológico de São Paulo, v.70, n.1, p.85-87, 2003.

BRAGANÇA, M.A.L.; De SOUZA, O.; ZANUNCIO, J.C. Environmental heterogeneity as a strategy for pest management in Eucalyptus plantations. Forest Ecology and Management v.102, p.9-12. 1998.

CORSEUIL, E.; SPECHT, A.; LANG, C. Saturniídeos (Lepidoptera: Saturniidae) registrados para o Rio Grande do Sul, Brasil. I. Hemileucinae. Biociências, v. 10, n.2, p.147-155, 2002.

DIAS, M. M. Estágios imaturos de Dirphia (Dirphia) moderata Bouvier, 1929 (Lepidoptera, Saturniidae). Revista Brasileira de Entomologia, v.32, n.2, p.273-278, 1988.

DYAR, H. G. The number of molts of lepidopterous larvae. Psyche, v.5, n.1, p.420-422, 1890.
GUEDES, R.N. et al. Species richness and fluctuation of defoliator Lepidoptera populations in Brazilian plantations of Eucalyptus grandis as affected by plant age and weather factors. Forest Ecology and Management, v.137, p.179-184. 2000.

HIGA, R. C. V. Eucalipto: Pesquisa amplia usos Espécies de Eucalyptus. Revista da Madeira, v.13, n.75, p.17, 2003.

HOLTZ, A. M. et al. Adaptação de Thyrinteina arnobia em novo hospedeiro e defesa induzida por herbívoros em eucalipto. Pesquisa Agropecuária Brasileira, v.38, n.4, p.453-458, $2003 a$.

HOLTZ, A. M. et al. Aspectos biológicos de Thyrinteina arnobia Lep.: Geometridae) provenientes de lagartas criadas em folhas de Eucalyptus cloeziana ou de Psidium guajava sob condições de campo. Revista Árvore, v.27, n.6, p.897-901, 2003b.

MAURICIO, R.; RAUSHER, M. D. Variation in the defense strategies of plants: are resistance and tolerance mutualy exclusive ? Ecology, v.78, n.4, p.1301-1311, 1997.

OLIVEIRA, H. N. et al. Rearing of Thyrinteina arnobia (Lepidoptera: Geometridae) on guava and eucalyptus in laboratory. Brazilian Archives of Biology and Technology, v.28, n.5, p.801-806, 2005.

PEREIRA, F.F. et al. Biological aspects of Dirphia moderata (Lepidoptera: Saturniidae) on Eucalyptus cloeziana and Psidium guajava. Brazilian Archives of Biology and Technology, v.51, n.2, p.369-372, 2008.

SANTOS, G. P.; ZANUNCIO, J. C.; ANJOS, N. A. Novos resultados da biologia de Psorocampa denticulata Schaus (Lepidoptera: Notodontidae), desfolhadora de eucalipto. Revista Árvore, v.6, n.2, p.121-132, 1982.

SANTOS, G. P.; ANJOS, N.; ZANUNCIO, J. C. Biologia de Hylesia nanus (Walker, 1855) (Lepidoptera: Attacidae), desfolhadora de cutieira (Joannesia princeps: Euphorbiaceae). Revista Ceres, v.35, n.201, p.479-485, 1988.

R. Árvore, Viçosa-MG, v.32, n.6, p.1119-1124, 2008 
SANTOS, G. P.; ANJOS, N.; ZANUNCIO, J. C. Biologia de Eustema sericea Schaus, 1922 (Lepidoptera: Notodontidae) desfolhadora de sobrasil, Colubrina rufa (Rhamnaceae). Anais da Sociedade Entomológica do Brasil, v.18, n.1, p.247-256, 1989.

SANTOS, G. P. et al. Biologia de Apatelodes sericea Schaus (Lepidoptera. Eupterotidae), desfolhador de eucalipto. Revista Árvore, v.9, n.2, p.171-179, 1985.

SANTOS, G. P. et al. Aspectos biológicos e morfológicos de Dirphiopsis eumedidoides (Vuillot, 1893) (Lepidoptera: Saturniidae) em folhas de Eucalyptus grandis. Revista Árvore, v.17, n.3, p.351-357, 1993.

SANTOS, G. P. et al. Biologia de Hylesia nanus (Walker) (Lepidoptera: Attacidae). Anais da Sociedade Entomológica do Brasil, v.25, n.3, p.479-482, 1996.

ZANUNCIO, J. C. et al. Levantamento e flutuação populacional de lepidópteros associados à eucaliptocultura: V- Região do Belo Oriente, Minas Gerais, junho de 1989 a maio de 1990.

Revista Árvore, v.14, n.1, p.35-44, 1990.

ZANUNCIO, J. C. Lepidópteros desfolhadores de Eucalipto: biologia, ecologia e controle. Viçosa, MG: Instituto de Pesquisas e Estudos Florestais; Sociedade de Investigações Florestais, 1993. 140p.
ZANUNCIO, J.C. et al. Levantamento e flutuação populacional de lepidópteros associados à eucaliptocultura: VI- Região de Belo Oriente, Minas Gerais. Pesquisa Agropecuária Brasileira, v.28, n.10, p.1121-1127.1993.

ZANUNCIO, J. C. et al. Alguns aspectos da biologia de Dirphia rosacordis (Lepidoptera: Saturniidae) em folhas de eucalipto. Revista Árvore, v.16, n.1, p.112-117, 1992.

ZANUNCIO, J.C. et al. Species richness and abundance of defoliating Lepidoptera associated with Eucalyptus grandis in Brazil and their response to plant age. Austral Ecology, v.26, n.6, p.582-589. 2001.

ZANUNCIO, T. V. et al. Caracterização das fases larval e adulta de Dirphia avicula (Lepidoptera: Saturniidae) em folhas de Eucalyptus urophylla. Revista Árvore, v.18, n.2, p.153-158, 1994.

ZANUNCIO, T.V. et al. Effect of plantation age on diversity and population fluctuation of Lepidoptera collected in Eucalyptus plantations in Brazil. Forest Ecology and Management, v.108, p.91-98. 1998. 